\title{
Miscibility and Phase Separation Behavior in Ternary Blends of Poly(vinylidene fluoride-hexafluoroacetone)/Poly(methyl methacrylate)/Poly(vinyl acetate)
}

\author{
S. Kobayashi, Jae Whan Cho, ${ }^{*}$ and Seizo Mryata \\ Department of Material Systems Engineering, Faculty of Technology, \\ Tokyo University of Agriculture and Technology, \\ Tokyo 184, Japan \\ * Department of Textile Engineering, College of Engineering, \\ Konkuk University, Seoul 133-701, Korea
}

(Received February 23, 1993)

\begin{abstract}
Miscibility of both binary and ternary blends between poly(vinylidene fluoridehexafluoroacetone) P(VDF-HFA), Poly(methyl methacrylate) (PMMA) and poly(vinyl acetate) (PVAc) was investigated by using dynamic mechanical and differential scanning calorimetric measurements. The interaction parameter in binary blends of P(VDF-HFA) and PMMA was -0.73 at $125^{\circ} \mathrm{C}$ and was largely affected by the existence of hexafluoroacetone units, compared with that of blends of poly(vinylidene fluoride) and PMMA. Phase separation behavior of this binary blends was elucidated by observing the change of transmittance on heating; the lower critical solution temperature (LCST) behavior was shown including both binodal and spinodal curves. Binary blends of P(VDF-HFA)/PVAc were also miscible and showed LCST behavior. Ternary blends of P(VDF-HFA)/PMMA/PVAc were miscible in spite of immiscible binary blends of PMMA/PVAc and showed LCST phase diagram. After phase separation, ternary miscible blends were separated to two phases of P(VDF-HFA)/PMMA with very small domains of PVAc and P(VDF-HFA)/PVAc with also very small PMMA. At higher temperature, phase separation composed of P(VDF-HFA)/PMMA and P(VDF-HFA)/PVAc occurred.

KEY WORDS Miscibility / Phase Separation / Ternary Blend / Interaction Parameter / Lower Critical Solution Temperature /
\end{abstract}

A considerable number of miscible polymer blends have been known though most pairs of polymers do not show mutual miscibility because of the small entropy of mixing for molecular chains. Moreover the miscibility in binary polymer blends is mostly interpreted in terms of the negative polymer-polymer interaction parameter ${ }^{1}$ and their miscible binary blends tend to phase separate at elevated or lower temperatures, which is called lower critical solution temperature (LCST) or upper critical solution temperature. ${ }^{2-4}$ However few studies on miscible ternary polymer blends have been reported since the last decade. They include poly(methyl methacrylate)/poly(epi- chlorohydrin)/poly(ethylene oxide) ${ }^{5}$ poly(vinylidene fluoride), PVDF/poly(methyl methacrylate)/poly(ethyl methacrylate), ${ }^{6}$ polystyrene/ polybutadiene/styrene-butadiene random or diblock copolymer, ${ }^{7}$ vinylidene chloride-vinylchloride copolymer/poly(vinyl chloride)/acrylonitrile-butadiene copolymer, ${ }^{8}$ and polycarbonate/poly(styrene-acrylonitrile)/aliphaticpolyester, ${ }^{4}$ etc. Miscible ternary blends can be enhanced by adding the third component which play a role of compatibilizer. The third component may be a type of copolymer of two other components or a different homopolymer as a compatabilizer of other immiscible pair.

The phase separation behavior for ternary 
mixtures has been often realized on the basis of Flory-Huggins theory. ${ }^{10}$ However reports on phase diagram in ternary polymer blends are very limited. This study describes the miscibility and phase separation behavior in ternary polymer blends consisting of different polymer species in the chemical structures, that is, poly(vinylidene fluoride-hexafluoroacetone) P(VDF-HFA), poly(methyl methacrylate) (PMMA) and poly(vinyl acetate) (PVAc); $\mathrm{P}(\mathrm{VDF}-\mathrm{HFA})^{11}$ is a crystalline polymer.

\section{EXPERIMENTAL}

\section{Sample Preparation}

Two kinds of P(VDF-HFA) were supplied by Central Glass Co. Two kinds of PMMA different in molecular weight and a kind of PVAc were supplied by Mitubishi Rayon Co. and Japan Synthetic Chemical Co., respectively. Their characteristics are given in Table I.

Binary and ternary blend films between P(VDF-HFA), PMMA, and PVAc were cast from solution of tetrahydrofuran at room temperature. They were dried in a vaccum drying oven at $80^{\circ} \mathrm{C}$ for 3 days and then at $150^{\circ} \mathrm{C}$ for 1 day. The thickness of samples obtained so was about $30-50 \mu \mathrm{m}$. Blend films were also cast using various other solvents of dimethylformaldehyde, dimethylacetamide, acetone, acetonitrile, and methyl ethyl ketone but no difference in the miscibility between their blend films was observed.

\section{Measurements}

Dynamic mechanical measurements were

Table I. Characteristics of polymers used in this study

\begin{tabular}{|c|c|c|c|c|}
\hline Polymer & $M_{n} \times 10^{5}$ & $M_{w} \times 10^{5}$ & $M_{w} / M_{n}$ & $\mathrm{~mol} \%$ \\
\hline PMMAI & 1.2 & 2.7 & 2.3 & \\
\hline PMMA2 & 0.4 & 0.95 & 2.4 & \\
\hline $\mathrm{P}(\mathrm{VDF}-\mathrm{HFA}) 1$ & 0.72 & 1.8 & 2.5 & $\begin{array}{c}91 / 9 \\
\text { VDF-HFA }\end{array}$ \\
\hline $\mathrm{P}(\mathrm{VDF}-\mathrm{HFA}) 2$ & 0.66 & 1.4 & 2.0 & $\begin{array}{c}92 / 9 \\
\text { VDF-HFA }\end{array}$ \\
\hline PVAc & 0.44 & - & - & \\
\hline
\end{tabular}

carried out to investigate the glass transition behavior by using the apparatus developed by Furukawa et al. ${ }^{12}$ Glass transition temperature $\left(T_{\mathrm{g}}\right)$ of sample was obtained from the maximum peak temperature of the curve of loss modulus versus temperature. The measurement was performed at $10 \mathrm{~Hz}$ at a heating rate of $2^{\circ} \mathrm{C} \mathrm{min}^{-1}$ and the sample was used in a size of length $8 \mathrm{~mm}$ and width $4 \mathrm{~mm}$.

Thermal properties were measured by using the differential calorimetry (Rigaku TG-DSC) with the sample of $5 \pm 1 \mathrm{mg}$ at a heating rate of $20^{\circ} \mathrm{C} \mathrm{min}^{-1}$. An equilibrium melting temperature was obtained from the extrapolation of linear plot between crystallization and melting temperatures.

The change of transmittance of samples on heating was observed by using the apparatus similar to the other's apparatus in principle. ${ }^{4}$ The transmittance of sample was measured by using a CdS of which electrical resistance changed with the intensity of an illuminated light through the sample. The electrical resistance of $\mathrm{CdS}$ was sensitive to the light having wavelength of $510-520 \mathrm{~nm}$ and was quite linear to the intensity of light. The output of CdS was recorded in a X-Y recorder with the temperature measured by a thermocouple on heating. The heating rate of $1^{\circ} \mathrm{C} \mathrm{min}^{-1}$ was used for all measurements and the transmittance behavior was not affected by the heating rate ranging from 0.5 to $4^{\circ} \mathrm{Cmin}^{-1}$.

\section{RESULTS AND DISCUSSION}

\section{Binary Blends}

Figure 1 shows the composition dependence of glass transition temperature of binary blends of P(VDF-HFA)1 and PMMA2. Only single $T_{\mathrm{g}}$ appeared in all binary blends throughout the entire composition range, indicating that P(VDF-HFA)1/PMMA2 binaries are miscible. Such a single $T_{\mathrm{g}}$ was observed in all the used blend samples containing P(VDF-HFA) and PMMA.

A melting depression of P(VDF-HFA) in 


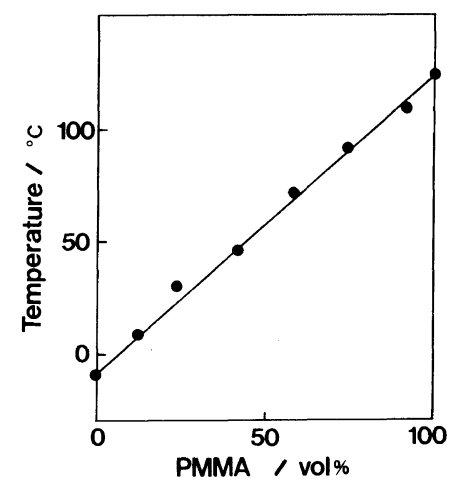

Figure 1. Composition dependence of glass transition temperatures $T_{\mathbf{g}}$ in binary blends of $\mathrm{P}(\mathrm{VDF}-\mathrm{HFA}) \mathrm{l}_{\text {/ }}^{\prime}$ PMMA2.

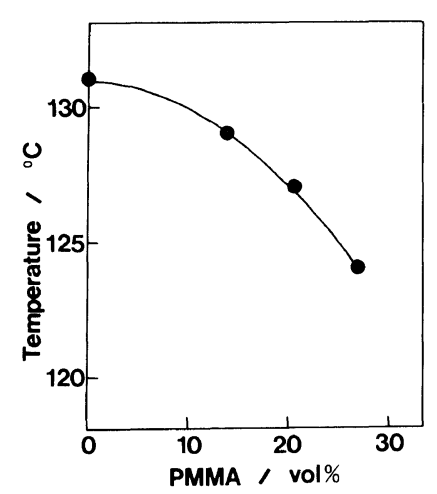

Figure 2. Composition dependence of equilibrium melting temperatures $T_{\mathrm{m}}{ }^{\circ}$ of binary blends of P(VDF-HFA)1/ PMMA2.

these miscible binary blends can yield some information about the polymer-polymer interaction parameter. Melting peaks were observed in the P(VDF-HFA)1/PMMA2 blends containing 60 weight percent of $\mathrm{P}(\mathrm{VDF}-\mathrm{HFA}) 1$ or more on DSC measurements. Figure 2 shows an equilibrium melting temperature which was obtained from the plot of melting versus crystallization temperatures, so-called HoffmanWeeks plot. ${ }^{13}$ The polymer-polymer interaction parameter, $\chi$ was calculated by using the following equation.

$$
\frac{1}{\phi_{1}}\left(\frac{1}{T_{\mathrm{m}}}-\frac{1}{T_{\mathrm{m}}{ }^{\circ}}\right)=-\chi \frac{R T}{\Delta H_{\mathrm{u}}} \frac{V_{2 \mathrm{u}}}{V_{1 \mathrm{u}}} \frac{\phi_{1}}{T_{\mathrm{m}}}
$$

Subscript 1 and 2 denote the amorphous and crystalline polymers, respectively and $T_{\mathrm{m}}$ and $T_{\mathrm{m}}{ }^{\circ}$ are the equilibrium melting temperatures of component 2 in the blend and the pure state, respectively. $\Delta H_{\mathrm{u}}$ is the heat of fusion per mole of repeating units in component $2, V_{u}$ is the molar volume of repeating units of component 2 and $\phi$ the volume fraction. Using $V_{1 \mathrm{u}}=84.0$ $\mathrm{cm}^{3} \mathrm{~mol}^{-1}, V_{2 \mathrm{u}}=41.9 \mathrm{~cm}^{3} \mathrm{~mol}^{-1}$, and $\Delta H_{\mathrm{u}}=$ $1.45 \mathrm{kcal} \mathrm{mol}^{-1}$, the interaction parameter between P(VDF-HFA)1 and PMMA2 was obtained to be -0.73 at $125^{\circ} \mathrm{C}$. It indicates the existence of a strong interaction between P(VDF-HFA) and PMMA, being in agreement with previous $T_{\mathrm{g}}$ results. A value of $\chi$ between PVDF and PMMA was reported to be -0.295 by $\mathrm{Nishi}^{14}$ and $-0.43 \sim-0.10$ by Wendroff ${ }^{15}$; the result by Wendroff represents the composition dependence of $\chi$ and the increase of $|\chi|$ with increasing concentration of PVDF. If the composition dependence of $\chi$ could be assumed in this study, $\chi=-0.73$ might be estimated more or less largely because only the samples containing $80 \mathrm{wt} \%$ of $\mathrm{P}(\mathrm{VDF}-\mathrm{HFA})$ or more were used for the calculation of equilibrium melting temperature. However, even regarding that the Wendroff's result is $\chi=-0.25$ for $80 / 20 \mathrm{wt} \%$ of PVDF/PMMA and -0.43 for $90 / 10 \mathrm{wt} \%$, the interaction between P(VDFHFA) and PMMA is stronger than that between PVDF/PMMA. It is regarded to be due to the miscibility between hexafluoroacetone units and PMMA or the repulsion effect ${ }^{21}$ in the random copolymer.

Miscible binary blends of P(VDF-HFA) and PMMA were transparent at room temperature throughout the entire composition range but exhibited turbidity above a critical temperature on heating. Figure 3 shows the typical patterns for the change of transmittance of blends with rising the temperature. Figure 3(a) exhibits the large changes of transmittance at $T_{\mathrm{b}}$ and $T_{\mathrm{w}}$, respectively. At two transition temperatures of $T_{\mathrm{b}}$ and $T_{\mathrm{w}}$, the change of color and turbidity of samples was observed; at $T_{\mathrm{b}}$, the original transparent sample changed partially white with a faint blue color and further heating up 


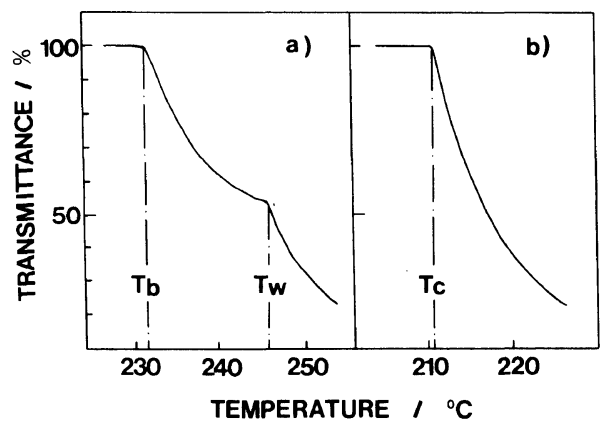

Figure 3. Changes of transmittance versus temperature in blends of P(VDF-HFA)2/PMMA2 on heating at a rate of $1^{\circ} \mathrm{C} \min ^{-1}$; (a) $10 / 90$, (b) $50 / 50 \mathrm{wt} \%$ of $\mathrm{P}(\mathrm{VDF}$ HFA)2/PMMA2.

to $T_{\mathrm{w}}$ or more made the sample fully turbid with white color. This change from transparence to white turbidity was reversible on heating or cooling through $T_{\mathrm{b}}$ or $T_{\mathrm{w}}$ and was observed for all the samples containing $40 \mathrm{wt} \%$ P(VDF-HFA) or less and $30 \mathrm{wt} \%$ PMMA or less. However for the samples containing $45-65 \mathrm{wt} \%$ of P(VDF-HFA), only one rapid drop of transmittance at $T_{\mathrm{c}}$ was observed as shown in Figure 3(b); the samples changed quickly from transparence to white turbidity at $T_{\mathrm{c}}$. These temperatures of $T_{\mathrm{b}}, T_{\mathrm{w}}$, and $T_{\mathrm{c}}$ in binary blends of P(VDF-HFA)1/PMMA1 were plotted versus blend composition as shown in Figure 4. It represents that the binary blends have the phase separation behavior of LCST type. ${ }^{4}$ In binary blends of P(VDFHFA)1/PMMA2, the LCST phase diagram was also obtained as shown in Figure 5. Blends with PMMA having smaller molecular weight exhibited the higher critical temperature in phase diagram and it is ascibed to the increase of mutual solubility between two polymer components owing to the lowering molecular weight of PMMA.

At this time, it would be suggested that the phase separation by the transmittance of blends is associated with nucleation and growth, and spinodal decomposition; in Figures 4 and 5, curves designated by vacant and filled circles are considered being corresponded to the

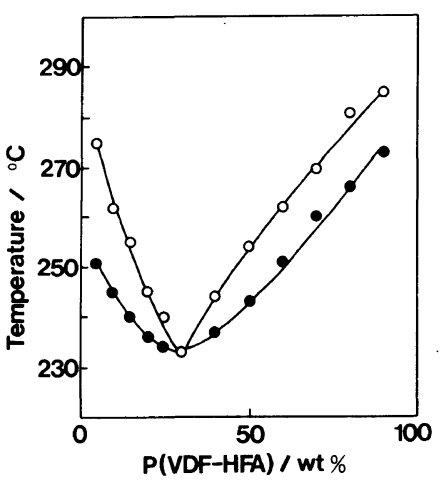

Figure 4. Phase diagram of binary blends of P(VDFHFA)1/PMMA1 in terms of cloud points: filled (O) and vacant $(\bigcirc)$ circles denote $T_{\mathrm{b}}$ and $T_{\mathrm{w}}$, respectively.

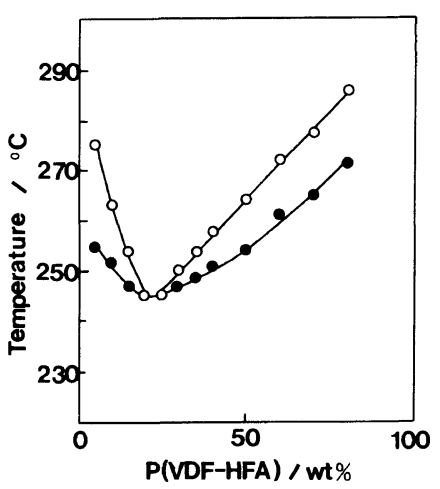

Figure 5. Phase diagram of binary blends of P(VDFHFA)1/PMMA2 in terms of cloud points; filled (O) and vacant $(O)$ circles denote $T_{\mathrm{b}}$ and $T_{\mathrm{w}}$, respectively.

spinodal and binodal curves, respectively. In order to clear it, the samples were annealed at $245^{\circ} \mathrm{C}$ for 1 hour, following the quenching into the mixture of water and ice. An optical microscopic observation of those samples led to the occurrence of the phase separation in spinodal region and their phase separated structure was very similar to the modulated structure of Cahn. ${ }^{16}$ The samples annealed in binodal region did not exhibit the phase separated structure which was observable in the samples annealed at $245^{\circ} \mathrm{C}$, though they took on the faint blue color in the visual observation. It indicates the existence of very small phase separated nucleus in binodal 
decomposition region. Therefore it is reasonable that two curves shown in phase separation are due to the spinodal and binodal, respectively.

On the other hand, the miscibility of binary blends of P(VDF-HFA) and PVAs was also investigated; these blends were also miscible over the entire composition range and showed LCST phase diagram. However according to Hasegawa, ${ }^{20}$ no phase separation in binary blend of P(VDF-HFA)/PVAc was observed within temperature range performed in the experiments. This difference of phase separation behavior is likely to result from difference of molecular weight of two pure components. Figure 6 shows $T_{\mathrm{g}}$ versus composition in binary

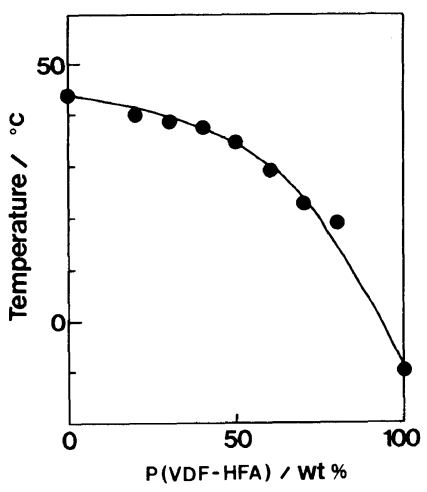

Figure 6. Blend composition dependence of glass transition temperature $T_{\mathrm{g}}$ of binary blends of P(VDFHFA)1/PVAc.

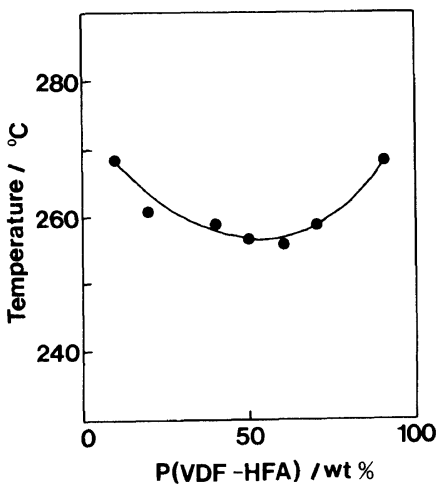

Figure 7. Phase diagram of binary blends of P(VDFHFA)2/PVAc in terms of cloud points. blends of P(VDF-HFA) 1 and PVAc. Single $T_{\mathrm{g}}$ was shown in all the blend samples though it was convex type to the blend composition, indicating the miscibility of blends. The phase diagram in blends of P(VDF-HFA) 1 and PVAc is shown in Figure 7; it takes a type of Figure 3(b). However binary blends of PMMA and PVAc showed the immiscibility over all the composition similarly as in other investigator's results. ${ }^{17}$

\section{Ternary Blends}

Figure 8 shows phase diagram in ternary blends of P(VDF-HFA)1/PMMA2/PVAc in terms of glass transition temperature. Miscibility exists between P(VDF-HFA) and PMMA molecules as well as between P(VDF-HFA) and PVAc, whereas PMMA and PVAc is not miscible. Single $T_{\mathrm{g}}$ was observed in ternary blends having a few of PMMA and rich PVAc regardless of blend composition of $\mathrm{P}(\mathrm{VDF}$ HFA), whereas double $T_{\mathrm{g}} \mathrm{s}$ were observed with an addition of rich PMMA. It indicates that ternary blends composed of P(VDF-HFA), PMMA and PVAc have the compatibility, on the basis of existence of only one $T_{\mathrm{g}}$ between $T_{\mathrm{g}} \mathrm{s}$ of pure components. Existence of single or double $T_{\mathrm{g}} \mathrm{s}$ dependent on blend composition of PMMA is related to the phase separation behavior in ternary blends. To investigate the phase separation behavior of ternary blends

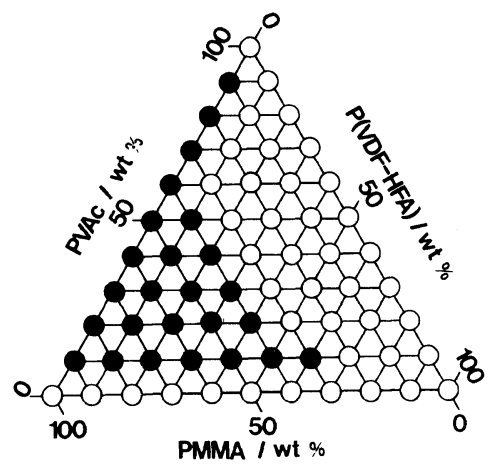

Figure 8. Ternary phase diagram of blends of P(VDFHFA)/PMMA/PVAc; vacant $(O)$ and filled $(O)$ circles denote the existence of single and double peaks in the loss modulus curve, respectively. 


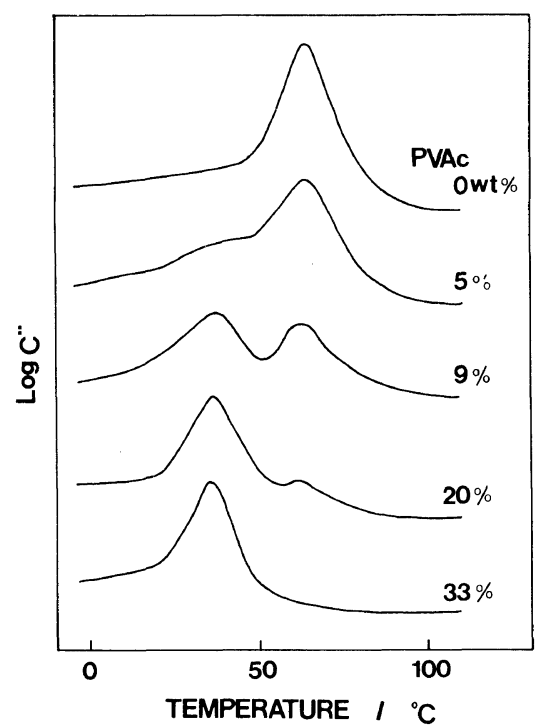

Figure 9. Temperature dependence of loss modulus $C^{\prime \prime}$ (arbitrary unit) in the ternary blends containing $1: 1: x$ P(VDF-HFA)/PMMA/PVAc by weight.

showing two glass transition temperatures in Figure 8 , the dynamic mechanical properties were measured in the samples having $1: 1: x$ of P(VDF-HFA)1/PMMA2/PVAc by weight, which is shown in Figure 9. Ternary blends containing 0 and $33 \mathrm{wt} \%$ of PVAc, respectively, that is, binary blend of $1: 1 \mathrm{P}(\mathrm{VDF}-\mathrm{HFA}) / \mathrm{PM}$ MA and ternary blend of $1: 1: 1$ P(VDFHFA)/PMMA/PVAc showed the single glass transition temperature at $66^{\circ} \mathrm{C}$ and $38^{\circ} \mathrm{C}$, respectively. It indicates the existence of only one phase in those blend samples. However ternary blends having $1: 1: 0.2-0.5 \mathrm{P}(\mathrm{VDF}$ HFA)/PMMA/PVAc showed still two glass transition peaks at $37-40^{\circ} \mathrm{C}$ and $62-65^{\circ} \mathrm{C}$ including a shoulder, respectively, indicating existence of two phases. The intensities of such two glass transition peaks varied relatively with the content of PVAc. It resumes that ternary blends having two glass transition temperatures exist in two phases. In spite of it, their measured refractive indices were near to the values calculated by assuming that the blends follow one phase model. It reflects that one of two phases exists in very small fraction or is

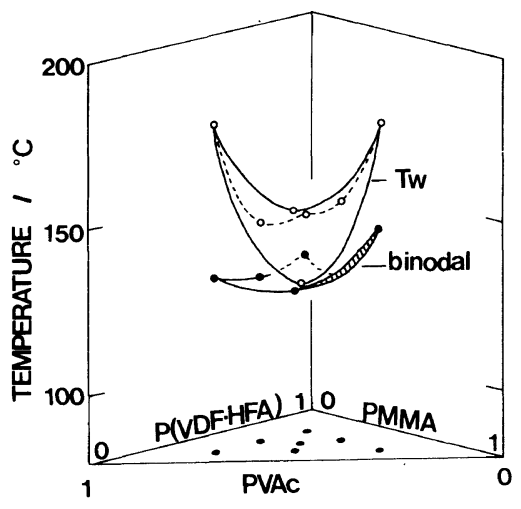

Figure 10. Phase diagram of ternary blends of P(VDFHFA)/PMMA/PVAc. The filled circles of the bottom plane denote the projected points of experimental data on the bottom.

dispersed in the other phase with a very small domain.

Phase separation behavior of ternary blends was also investigated by measuring the change of transmittance on heating similarly as in previous binary blends. Figure 10 shows the ternary phase diagram of P(VDF-HFA)1/PMMA2/ PVAc. Ternary blends exhibit LCST phase diagram that critical point is located near the center of ternary blend composition with the sharply curved spinodal and the smoothly curved binodal. However the reversability of phase separation in ternary blends was not observed on heating or cooling, dissimilarly to the case in binary blends of P(VDF-HFA)/ PMMA. It may be due to too slow phase separation because of the difference of interaction parameters between blend components.

Figure 11 shows the temperature dependence of loss modulus in ternary blends. An unannealed sample exhibited a single $T_{\mathrm{g}}$ but the sample annealed at $180^{\circ} \mathrm{C}$ which was above phase separation temperature exhibited two peaks of the main peak at $33^{\circ} \mathrm{C}$ and the shoulder at $80-90^{\circ} \mathrm{C}$. It means that the phase separation of ternary blends occurred in two phases. For the samples annealed at $250^{\circ} \mathrm{C}$ which is above phase separation temperature 


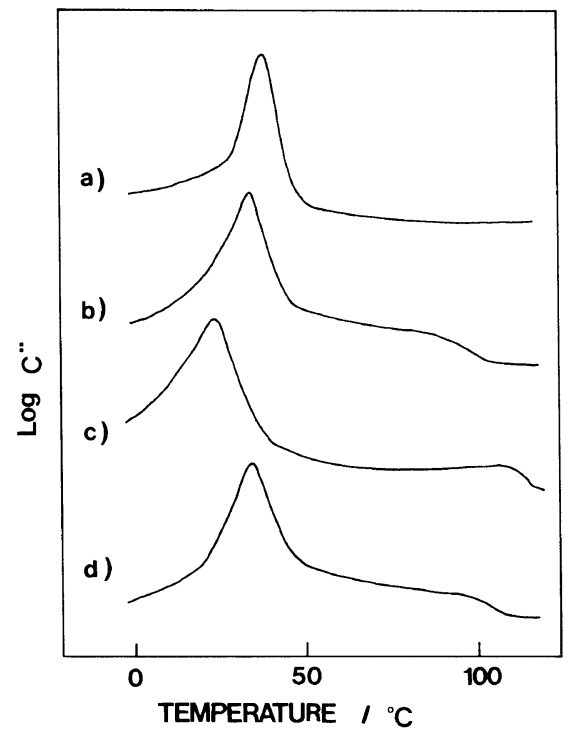

Figure 11. Temperature dependence of loss modulus $C^{\prime \prime}$ (arbitrary unit) of ternary blends of $0.4: 1: 1 \mathrm{wt} \%$ P(VDF-HFA)/PMMA/PVAc annealed at various temperatures: (a), unannealed; (b), annealed at $180^{\circ} \mathrm{C}$ for 1 hour; (c), annealed at $250^{\circ} \mathrm{C}$ for 1 hour, following the quenching into liquid nitrogen; (d), annealed at $180^{\circ} \mathrm{C}$ for 1 hour after heat treatment of (c).

of binary blends of P(VDF-HFA)/PMMA and below that of P(VDF-HFA)/PVAc, main peak temperature was shifted toward lower temperature of $23^{\circ} \mathrm{C}$ and higher temperature of $110^{\circ} \mathrm{C}$, respectively, compared with the results of Figure 11(b). Further annealing the sample shown in Figure $11(\mathrm{~b})$ at $180^{\circ} \mathrm{C}$ gives the same relaxational curve as that of Figure 11(b). It reflects that the phases of miscible ternary blends are separated to two phases of P(VDF-HFA)/PMMA with small PVAc domains and P(VDF-HFA)/PVAc with very small PMMA one. But it is regarded that above phase separation temperature, the phase separation results in the existence of two phases of P(VDF-HFA)/PMMA and P(VDF-HFA)/ PVAc.

After all, ternary blends of P(VDF-HFA)/ PMMA/PVAc have the similar phase separation behavior which can be observed in binary blends of P(VDF-HFA)/PMMA. To analyze phase separation behavior of ternary blends

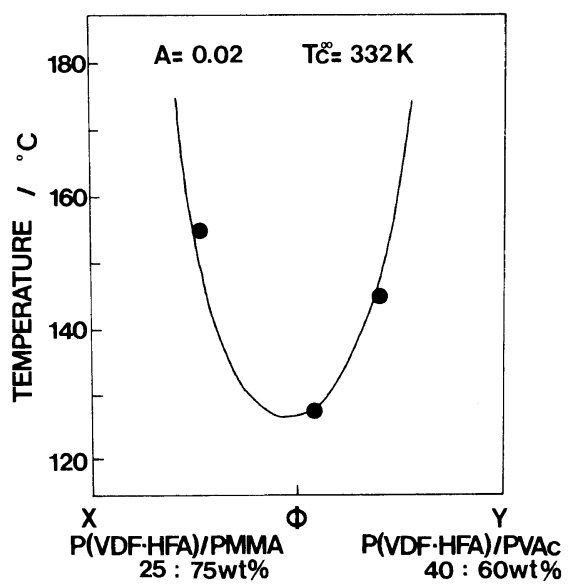

Figure 12. Spinodal curve obtained from the experimental data ( ) and the calculation (-). $\phi$ denotes the volume fraction of $\mathrm{Y}$ component in ternary blends separated to two phases of P(VDF-HFA)/PMMA (X) ratio of $25 / 75$ and $\mathrm{P}(\mathrm{VDF}-\mathrm{HFA}) / \mathrm{PVAc}(\mathrm{Y})$ ratio of $40 / 60$ by weight.

more reasonably, eq 2 was applied to this blend system. ${ }^{18}$ This equation is used to elucidate the phase separation behavior of binary blends with the correlation between calculated spinodal curve and the cloud points. Here we will resume that the phase separation of ternary blends at high temperature occurs in two domains by applying ternary blends to this equation.

$$
\frac{1}{T_{\mathrm{sp}}}=\frac{1}{T_{\mathrm{c}}^{\infty}}\left[1-\frac{1}{2 A}\left(\frac{1}{\phi_{1, \mathrm{sp}} m_{1}}+\frac{1}{\phi_{2, \mathrm{sp}} m_{2}}\right)\right]
$$

where 1 and 2 designates the phases of P(VDFHFA)/PMMA and P(VDF-HFA)/PVAc, respectively and $m_{i}$ is the degree of polymerization of $i$ component, $\phi_{i}$ volume fraction of $i$ component, $T_{\mathrm{c}}^{\infty}$ the phase separation temperature at infinite molecular weight, and $A$ is a constant corresponded to a entropic term of polymer-polymer interaction parameter. The blend composition of components 1 and 2 after phase separation of ternary blends was calculated from the location of peak temperature of loss modulus curve versus temperature. The calculated and experimental spinodal curves are shown in Figure 12. From the fitting 
of the experimental data to the theoretical curve, $A=0.02$ and $T_{\mathrm{c}}^{\infty}=332 \mathrm{~K}$ were obtained. The value of $A=0.02$ in this study is near to $A=0.016$ of binary blend of Polystyrene, PS and poly(vinyl metyl ether) (PVME) by other author. ${ }^{19} T_{\mathrm{c}}$ and $T_{\mathrm{c}}^{\infty}$ in this study represents a difference of $69^{\circ} \mathrm{C}$ and it is nearly same value as $70^{\circ} \mathrm{C}$ of blend of PS/PVME. Consequently it can be said that the phase separation in the ternary blends of P(VDF-HFA)/PMMA/PVAc at high temperature arouses two domains of P(VDF-HFA)/PMMA and P(VDF-HFA)/PVAc.

\section{CONCLUSIONS}

Binary blends of P(VDF-HFA)/PMMA showed the complete miscibility and their interaction parameter was -0.73 at $125^{\circ} \mathrm{C}$. LCST phase behavior of this blend could be elucidated by observing the change of transmittance on heating the samples. Existence of hexafluoroacetone units in P(VDF-HFA) gave the stronger interaction to PMMA and lowered the critical temperature in the LCST phase diagram, compared with binary blend of PVDF/PMMA. Binary blends of P(VDFHFA)/PVAc were also miscible and showed LCST behavior.

Ternary blends of P(VDF-HFA)/PMMA/ PVAc were miscible even through binary blends of PMMA/PVAc were immiscible. Existence of P(VDF-HFA) in ternary blends led to the lowering of critical temperature of LCST phase diagram and it was regarded as an effect of the interaction parameter. After phase separation, ternary blends were divided in two phases of P(VDF-HFA)/PMMA with very small PVAc domains and P(VDFHFA)/PVAc with very small PMMA and at higher temperature, there existed two phases of P(VDF-HFA)/PMMA and P(VDF-HFA)/ PVAc.

\section{REFERENCES}

1. M. M. Coleman, J. F. Graf, and P. C. Painter, "Specific Interactions and the Miscibility of Polymer Blends," Technomic Publishing Company, Lancaster, PA, 1991.

2. T. Nishi, J. Macromol. Sci.-Phys., B17(3), 517 (1980).

3. D. R. Paul and S. Newman, Ed., "Polymer Blends," Vols. I \& II, Academic Press, London, 1978.

4. R. E. Bernstein, C. A. Cruz, D. R. Paul, and J. W. Barlow, Macromolecules, 10, 681 (1977).

5. K. E. Min, J. S. Chiou, J. W. Barlow, and D. R. Paul, Macromolecules, 28, 1721 (1987).

6. T. K. Kwei, H. L. Frisch, W. Radigan, and S. Vogel, Macromolecules, 10, 157 (1977).

7. D. Rigby, J. L. Lin, and R. J. Roe, Macromolecules, 18, 2269 (1985).

8. Y. Y. Wang and S. A. Chen, Polym. Eng. Sci., 21(1), 47 (1981).

9. V. S. Shah, J. D. Keitz, D. R. Paul, and J. W. Barlow, J. Appl. Polym. Sci., 32, 3863 (1986).

10. T. Nishi and T. T. Wang, Macromolecules, 8, 909 (1975).

11. V. L. Maksimov, L. I. Tarutina, N. A. Dreiman, T. S. Dunayevskaya, and L. N. Pirozhnaya, Vysokomol. Soyed., Ser. A, 17(7), 1585 (1975).

12. T. Furukawa and E. Fukuda, J. Polym. Sci., Polym. Phys. Ed., 16, 92 (1978).

13. J. D. Hoffman and J. J. Weeks, J. Res. Natl. Bur. Stand., 66, 13 (1962).

14. P. J. Flory, "Principles of Polymer Chemistry," Cornell University Press, Ithaca, New York, N.Y., 1953.

15. J. H. Wendorff, J. Polym. Sci., Polym. Lett. Ed., 18, 439 (1980).

16. J. W. Cahn, J. Chem. Phys., 42, 93 (1965).

17. S. Ichihara, A. Komatsu, and T. Hata, Polym. J., 2, 640 (1971).

18. T. Nishi, J. Macromol. Sci. Phys., B17, 517 (1980).

19. T. Nishi, Rep. Prog. Polym. Phys. Jpn., 20, 225 (1977).

20. M. Hasegawa and S. Akiyama, Polym. J., 20, 471 (1988).

21. G. ten Brinkle, F. E. Karasz, and W. J. MacKnight, Macromolecules, 16, 1827 (1983). 